\title{
Transfert dans l'acquisition des expressions idiomatiques en français langue étrangère
}

\author{
Luz Dary Cáceres-Guerrero*
}

Master en didactique du français langue étrangère. Enseignant-chercheur,

Universidad Industrial de Santander, Bucaramanga, Colombie.

Courrier électronique:

luzdary9001@hotmail.com.

Recibido: 21 de mayo del 2017

Aprobado: 1 de noviembre del 2017

Cómo citar este artículo: Cáceres-Guerrero, Luz Dary. "Transfert dans l'acquisition des expressions idiomatiques en français langue étrangère". Rastros Rostros 19.35 (2017): 1-15. Web. doi: https://doi. org/10.16925/2382-4921.2017.35.06

\section{Résumé}

Introduction: Cette recherche examine dans quelle mesure les apprenants non-natifs utilisent la connaissance de leur L1 afin de comprendre et de produire les expressions idiomatiques de la L2. Méthodologie: Vingt-six Colombiens apprenants de français (niveau B2) et vingt-six hispanophones vivant à Toulouse (niveau $\mathrm{C} 1$ ) ont participé à cette étude. Trente expressions idiomatiques du français ont été testées, classées en trois catégories : a) identiques en forme et en sens à une expression équivalente en espagnol, b) similaires à une expression équivalente en espagnol, c) différentes à une expression équivalente en espagnol. Quatre tests ont été administrés: deux tests de production, un test d'acceptabilité et un test de compréhension. Résultats: Pour vérifier l'hypothèse du transfert linguistique selon laquelle les expressions identiques sont faciles à comprendre et à produire, alors que les expressions différentes présentent plus de difficultés dans les deux compétences (compréhension et production), la catégorie des expressions similaires viendrait ainsi nuancer l'hypothèse du transfert et ce, notamment en termes d'opacité sémantique, de fréquence, de structure et de lexique. Conclusions: Les propositions didactiques en cours de Français langue étrangère (FLE) devraient partir de ce qui est connu par les apprenants pour, au fur et à mesure de leurs processus d'acquisition de la L2, aborder les particularités ou les différences entre la L1 et la L2.

Mots clés: acquisition, expressions idiomatiques, Français langue étrangère, transfert linguistique. 


\title{
Transferencia en la adquisición de expresiones idiomáticas en francés como lengua extranjera
}

\section{Resumen}

Introducción: esta investigación examina hasta qué punto los estudiantes no nativos utilizan el conocimiento de su L1 para comprender y producir expresiones idiomáticas de la L2. Metodología: veintiséis estudiantes colombianos de francés (nivel B2) y veintiséis hispanos que viven en Toulouse (nivel C1) participaron en este estudio. Se evaluaron treinta expresiones idiomáticas del francés, clasificadas en tres categorías: a) idénticas en forma y significado a una expresión equivalente en español, b) similares a una expresión equivalente en español, c) diferentes a una expresión equivalente en español. Se realizaron cuatro pruebas: dos pruebas de producción, una prueba de aceptabilidad y una prueba de comprensión. Resultados: para probar la hipótesis de transferencia lingüística de que las expresiones idénticas son fáciles de entender y de producir, mientras que las diferentes expresiones presentan más dificultades en ambas habilidades (comprensión y producción), la categoría de expresiones similares matizaría así la hipótesis de la transferencia y esto, en particular en términos de opacidad semántica, frecuencia, estructura y léxico. Conclusiones: las propuestas didácticas en francés como idioma extranjero (FLE) deben comenzar a partir de lo que los alumnos saben para que, a medida que su proceso de adquisición de L2, aborden las particularidades o diferencias entre el L1 y la L2.

Palabras clave: adquisición, expresiones idiomáticas, francés como lengua extranjera, transferencia lingüística.

\section{Transfer in the acquisition of idiomatic expressions in French as a Foreign Language}

\begin{abstract}
Introduction: this investigation examines to what extent non-native students use the knowledge of their first language (L1) to understand and produce idiomatic expressions in the second language (L2). Methodology: twenty six Colombian students of French (Level B2) and twenty six Hispanic students living in Toulouse (level C1) took part in this study. Thirty idiomatic expressions classified in three categories were evaluated: a) similar in form and meaning to an equivalent expression in Spanish, b) similar to an equivalent expression in Spanish, c) different from an equivalent expression in Spanish. Four tests were made: two production tests, a reception test and a comprehension test. Results: to test the linguistic transfer hypothesis that identical expressions are easy to understand and produce, while the different expressions present more difficulties in both skills (comprehension and production), the category of similar expressions would thus nuance the transfer hypothesis, and that, particularly in terms of semantic opacity, frequency, structure and lexicon. Conclusion: the didactic approach in French as Foreign Language (FFL) must start from what the students know so that as part of their process of L2 acquisition, they address the particularities and differences between L1 and L2.
\end{abstract}

Key words: acquisition, idiomatic expressions. French as a Foreign Language, linguistic transfer 


\section{Introduction}

L'enseignement-apprentissage des expressions idiomatiques (EI) auprès d'étudiants non-natifs est considérée actuellement comme l'une des préoccupations au sein des travaux de recherche dans le cadre de la didactique des langues, car l'apprentissage d'une langue vivante demande l'appropriation non seulement des structures grammaticales et syntaxiques mais aussi de la connaissance de sa phraséologie.

Cette problématique liée au domaine de la phraséodidactique nous a apparue intéressante en raison de deux constats : en premier lieu, les tournures idiomatiques sont des suites qui exigent l'appropriation de toutes les dimensions du système linguistique, ce qui permet d'inférer que la bonne maîtrise de ces tournures est une marque d'un niveau linguistique très élevé dans une L2. Pourtant, l'appropriation de ces suites figées de la langue représente un très haut degré de complexité pour les locuteurs non-natifs au niveau de la compréhension et de la production dans la L2.

En deuxième lieu, si les EI d'une L2 sont difficiles à acquérir pour les apprenants étrangers et même pour les niveaux avancés, c'est entre autres raisons parce que ces tournures méritent d'occuper une place centrale au sein de l'enseignement des langues étrangères. Mais cette complexité est également due au registre des EI qui varie selon le contexte d'énonciation et aussi selon l'interprétation littérale ou figurée de la tournure; ces facteurs constituent une difficulté supplémentaire pour l'apprenant surtout lorsqu'il n'a aucune familiarité avec l'EI.

Or, les lacunes des apprenants se révèlent lorsqu'ils se heurtent, aussi bien à l'oral qu'à l'écrit, à des EI très opaques dans la L2. Dans cette perspective, l'apprenant essaie de pallier sa méconnaissance idiomatique en faisant des transferts positifs ou négatifs entre la L1 et la L2. Concernant les transferts, le transfert positif pourrait aider considérablement l'évolution de l'interlangue de l'apprenant; en revanche le transfert négatif représente un obstacle dans la cohérence et la cohésion discursive, ce qui risque d'être une rupture dans l'efficacité de la communication lorsque l'apprenant ne dispose pas d'une riche connaissance des dissemblances entre les deux systèmes linguistiques.

En ce sens, notre travail, encadré par des différentes théories de la recherche telles que la psycholinguistique, la linguistique contrastive, la phraséologie et les conceptions stipulées dans le champ de la didactique des langues étrangères, a pour objectif de déterminer dans quelle mesure les apprenants emploient la connaissance de leur L1 afin de comprendre et de produire les EI de la L2. Pour ce faire, nous avons élaboré deux tests de production, un test d'acceptabilité et un test de compréhension afin d'évaluer deux publics différents : des hispanophones (Latino-Américains) vivant à Toulouse, France, et des hispanophones de la Colombie.

Dans cette perspective, nous proposerons trois catégories parmi les EI (identiques, similaires et différentes) afin d'analyser quelle catégorie est susceptible d'avoir le plus de cas de transfert positif ou de transfert négatif. De plus, notre travail se propose de vérifier si le degré de transparence sémantique dans les images phraséologiques et la connexion interlinguistique influencent la compréhension et la production des EI et si le niveau de langue est déterminant pour la compréhension et la production des tournures idiomatiques d'une manière efficace.

Ainsi, l'intérêt initial de cette étude concerne, un premier lieu, une approche théorique dans le domaine de la phraséologie et la phraséodidactique. Ensuite, il est nécessaire d'aborder les similitudes et les différences interlinguistiques afin de bien définir la théorie du transfert linguistique en ce qui concerne l'enseignement-apprentissage des EI. Enfin, nous expliquerons et détaillerons notre procédure méthodologique afin de nous pencher sur les résultats obtenus, l'analyse respective et la discussion à propos de nos résultats de recherche.

Pour commencer, la phraséologie est un terme qui concerne l'analyse d'expressions figées propres à une langue donnée. Elle est considérée, au sein de la linguistique, comme une discipline autonome qui englobe les caractéristiques des séquences lexicales perçues comme préconstruites et souvent utilisées dans la vie courante. L'originalité de ce type de langage, qu'on retrouve à l'oral et à l'écrit, repose sur la présence d'éléments polylexicaux, c'est-à-dire, de séquences qui sont composées de plusieurs mots, qui à l'origine étaient autonomes, tout en formant des structures unitaires, ce qui permet de les interpréter dans leur ensemble (Gonzalez 16). En effet, les premières allusions évoquées à ce sujet remontent à l'époque de Ferdinand de Saussure, dans son Cours de linguistique générale. Il fait référence, à la notion de liberté des syntagmes; si les syntagmes étaient formés avec des combinaisons libres (comme la phrase), ils appartiendraient à la parole. Pourtant, il existe des unités complexes qui ne sont pas des syntagmes 
libres, mais sont déjà construites et sont le résultat de la tradition culturelle (172).

De même, Charly Bally, disciple de Saussure, a approfondi sur quelques théories entres elles l'analyse des EI dont il fait une étude très minutieuse, plus particulièrement dans son ouvrage Traité de stylistique française. L'auteur remarque que "dans la langue maternelle, l'assimilation des faits de langage se fait surtout par les associations et les groupements dans lesquels l'esprit fait entrer les mots. " (66) Certains groupements peuvent être passagers, mais il y en a d'autres qui sont stables grâce à l'usage courant de ces unités par les locuteurs, ce qui permet de former des unités indissolubles lesquelles s'appellent locutions phraséologiques.

Ces deux théories que nous avons considérées ont un caractère important pour l'insertion et l'analyse des expressions idiomatiques, car grâce à la présence de ces éléments incontournables dans le discours, il faut leur accorder un statut central dans toutes les théories linguistiques. Pour ce faire, selon Capra, l'analyse des EI ne peut pas être uniquement l'objet d'un regard syntaxique, mais ces tournures méritent d'être abordées pour leur richesse sémantique et pour leur caractère polysémique. En effet, les études faites au sein de la phraséologie se font sentir dans le domaine de la didactique de langues étrangères. C'est ainsi que la phraséodidactique a cherché sa place centrale dans le processus d'enseignement-apprentissage de langues.

La phraséodidactique est, donc, une discipline qui concerne l'enseignement-apprentissage des expressions figées telles que les collocations, les parémies et l'EI. Son objectif principal est de revendiquer un traitement spécifique pour implémenter des techniques visant l'acquisition chez l'apprenant d'une compétence nécessaire en expressions figées, car la maîtrise de ces tournures " est très importante pour l'intelligence d'une langue étrangère. Inversement, l'emploi de séries incorrectes est un indice auquel on reconnaît qu'un étranger est peu avancé dans le maniement de la langue ou qu'il l'a apprise mécaniquement» (Bally 73).

En ce sens, depuis une vingtaine d'années, les linguistes et les didacticiens ont reconnu l'importance de créer des manuels et des outils méthodologiques pour l'enseignement-apprentissage d'unités phraséologiques en classe de langue afin de développer chez l'apprenant la compétence communicative, socioculturelle, métaphorique et pragmatique. Ainsi, le maniement correct des EI permet de s'exprimer de façon précise et naturelle dans la langue étrangère (Bolly 25). En effet, pour bien maîtriser une langue étrangère, il est nécessaire de produire et de comprendre un discours où l'emploi d'EI engendre une compétence de haut niveau dans l'intercommunication (Théophanous 125). En conséquence, selon Hoa, une action méthodologique, pédagogique et didactique efficace doit s'orienter vers l'approfondissement d'éléments phraséologiques de la langue.

Les EI en tant que groupements complexes exige un traitement rigoureux et systématique dans les unités pédagogiques, car il ne suffit pas seulement d'enseigner le lexique simple, tel que chat, homme, arbre, livre, etc., il faut aussi apprendre les expressions figées de la langue en soulignant celles qui ont un niveau d'opacité très élevé (Gonzalez) ; par exemple : poser un lapin, donner la langue aux chats, il y a anguille sous roche, etc. Nous avons en effet le sentiment que l'intention principale de l'enseignement est de faire découvrir aux apprenants le contenu implicite des EI pour que dans l'échange communicatif ils ne soient pas confrontés à des malentendus; et pour ce faire, il nous faudra sans doute renouveler les méthodes d'enseignement en accordant une place centrale à la phraséologie en tenant compte la compréhension et la production.

De cette manière, l'introduction des EI doit être considérée dès les premiers niveaux d'apprentissage d'une langue étrangère. Le but serait de parvenir à mieux sensibiliser l'apprenant aux procédés d'emploi de ces tournures signifiantes (Guilhermina 129). Dès le premier contact avec la langue étrangère, l'apprenant se rend compte des différences et similitudes lexicales entre la L2 et celles de la L1. Lapprenant remarque, à travers l'apprentissage des EI, les relations existantes au niveau sémantique, syntactique, ainsi que l'évocation d'un contraste d'ordre culturel ; en ce sens, le processus d'acquisition permet d'explorer au maximum l'aspect interculturel des éléments phraséologiques favorisant un système cohérent dans l'apprentissage, de tendre par ce biais à ce que les savoirs deviennent des savoir-faire (Théophanous 176).

Pour ce qui relève de la production et de la compréhension des EI, selon Gibbs et O'brien, il faudrait souligner que pour un apprenant étranger, il est plus facile de décoder que d'encoder ou produire de manière adéquate un énoncé qui ait une tournure idiomatique. Au moment où l'apprenant emploie une expression, il doit tenir compte du caractère stable et invariable de chaque constituant. Dans l'exemple donner sa langue au chat, il est impossible de changer 
chat pour chien ou langue pour dents. Cette spécificité des EI ne peut pas être laissée de côté dans l'enseignement des langues. Au contraire, elle doit être abordée en considérant le niveau des apprenants, c'est-à-dire que l'enseignant doit choisir les EI selon leur degré d'opacité en tenant compte des compétences des apprenants et aussi de la langue maternelle (Detry, Estratégias memorísticas 23).

\section{L'hypothèse du transfert linguistique : les similitudes et les différences}

Le facteur interlinguistique (Cummins et Swain ; Ellis) correspond à la manière dont cet aspect est le résultat des similitudes et des différences grâce au contact entre les structures linguistiques des langues au niveau phonétique, phonologique, morphologique, syntaxique ou lexical.

Concernant le processus d'enseignement-apprentissage, ce facteur peut inférer irrémédiablement, soit de façon négative, soit de manière positive, dans le décodage et l'encodage des unités phraséologiques. Nous pensons en effet que dans l'enseignement-apprentissage d'une L2, il est nécessaire de souligner les similarités syntaxiques et sémantiques entre les deux systèmes linguistiques. De plus, l'apprenant aura besoin de connaître les différences lexicales et surtout sémantiques d'une langue à une autre, car chaque système dispose de ses propres structures figées lesquelles sont intimement liées à la convention culturelle.

Dans cette perspective, cet aspect interlinguistique peut affecter de manière positive la compréhension quand l'image phraséologique dans la L2 trouve son équivalent sémantique et syntaxique dans la L1. Par exemple, pour un apprenant hispanophone, l'expression telle que prendre le taureau par les cornes lui serait plus facile à décoder dans son sens sous-jacent, car cette expression trouve son équivalence sémantique et syntaxique en espagnol : tomar/coger el toro por los cuernos/cachos. Les correspondances dans les deux langues ou en quelque sorte le transfert que l'apprenant opère lui permettrait d'accéder directement au sens idiomatique de l'expression, ce qui exigerait de lui moins de temps de réflexion lors de la décomposition lexicale pour envisager l'image littérale et ensuite la formation du sens figuré.

Par contre, pour un allophone, il serait plus complexe de décoder l'expression française faire d'une pierre deux coups, car l'expression anglaise To kill two birds with one stone ne correspond pas dans sa formation lexicale à celle du français. Néanmoins l'apprenant hispanophone qui étudie l'anglais, trouvera possiblement plus de rapprochement entre l'expression espagnole et l'expression anglaise, car matar dos pájaros de un tiro renvoie à la même correspondance lexicale entre les deux langues, ce qui lui permettrait de visualiser plus facilement l'image phraséologique. Ainsi, les correspondances lexicales et aussi syntactiques entre les langues aident l'apprenant à mieux comprendre l'expression étrangère en lui permettant de vaincre l'opacité de la tournure. «Por lo tanto, la referencia a la LM puede ayudar, en ciertos casos, a los alumnos a vencer la opacidad intralingüística de la EIE, al darle la posibilidad de reestablecer un cierta transparencia de tipo interlingüístico.» ${ }^{1}(\mathrm{De}-$ try, Estrategias memorísticas 74)

Cependant, il existe un autre facteur qui pourrait intervenir dans la compréhension des EI : il s'agit des " petites divergences lexicales " rencontrées entre la L1 et la L2. Celles-ci peuvent interférer dans la structure figée de la tournure, car le changement d'un syntagme pourrait affecter la compréhension de la part d'un interlocuteur natif ou compromettre la maîtrise de la tournure. Ainsi, nous trouvons des expressions presque identiques dans les langues, mais dont les constitutifs varient dans leur forme lexicale. Par exemple, l'expression française coûter les yeux de la tête, se dit en espagnol costar un ojo de la cara (coûter un oeil du visage), et en anglais It costs an arm and a leg (coûter un bras et une jambe).

Dans ces exemples, nous pouvons remarquer que dans les trois langues les tournures renvoient à un unique champ sémantique, mais il y a des divergences au niveau lexical: en espagnol, les locuteurs emploient un oeil et le visage tandis qu'en anglais ils emploient le bras et la jambe au lieu des yeux et la tête en français. Donc, ces similitudes interlinguistiques pourraient amener l'apprenant à faire des substitutions d'un syntagme de l'expression étrangère pour un autre de la langue maternelle. Dans ce cas-là la tournure n'aurait pas la même force expressive que celle qui est formée par les constitutifs conventionnels.

Par ailleurs, les similitudes interlinguistiques peuvent aussi amener le locuteur non-natif à faire des associations erronées de l'interprétation du sens figuré de la tournure étrangère, car dans les deux

[Traduction libre] : En conséquence, la référence à la LM peut aider, dans certains cas, aux apprenants à vaincre l'opacité intralinguistique de l'EIE en donnant l'occasion de rétablir une certaine transparence de type interlinguistique. 
langues, l'image apparaît comme identique. Par exemple, dans la phrase suivante Marie ne peut pas parler à sa maman de ses problèmes, car elle a les nerfs à fleur de peau en ce moment, l'apprenant hispanophone aura tendance à interpréter l'expression en l'assignant de la même signification que de la tournure en espagnol, car il va trouver que l'expression corresponde dans sa formation lexicale à celle de sa L1 (tener los nervios a flor de piel).

Cependant la signification de la tournure française fait référence à quelqu'un qui est facilement irritable, plutôt sensible, tandis que celle de l'espagnol renvoie à une connotation d'inquiétude ou de peur de faire quelque chose, comme dans la phrase suivante : Luis tiene los nervios a flor de piel porque va a presentar el examen (Louis a les nerfs à fleur de peau parce qu'il va présenter l'examen). De la même manière, nous trouvons l'expression française avoir un poil dans la main dont la correspondance en espagnol est tener pelos en la mano. Les deux tournures coïncident dans la concordance des constitutifs, mais l'expression française est employée pour indiquer que quelqu'un est paresseux ou "fainéant ", tandis que l'expression espagnole renvoie au registre du vulgaire et est employée pour dire que quelqu'un se masturbe habituellement.

Pour aller plus loin dans l'interprétation erronée des EI, il faut se pencher sur les métaphores qui sont identiques ou similaires dans la L2 et dans L1, mais qui renvoient à une signification totalement différente dans les deux langues. Par exemple, la tournure française Avoir le coeur sur la main signifie « quelqu'un de généreux ». L'apprenant hispanophone pourrait l'associer avec tener el corazón en la mano, car elle est formée avec les mêmes éléments lexicaux que ceux $\mathrm{du}$ français. Pourtant, la tournure espagnole signifie «quelqu’un qui parle sincèrement ». Néanmoins, le locuteur hispanophone essaiera d'attribuer certaines métaphores de la L1 à la L2, ainsi il trouvera les tournures espagnoles suivantes : tener el corazón en la boca (avoir le coeur dans la bouche) pour indiquer que "quelqu'un a peur d'affronter quelque chose» ou ponerse la mano en el corazón (se mettre la main sur le coeur) pour demander à " quelqu'un d'avoir de la compassion pour quelqu'un d'autre ».

Dans cette perspective, il est nécessaire de souligner le rôle que jouent les similitudes et les différences interlinguistiques, notamment entre la L2 et la L1, dans le processus d'inférence tout d'abord de l'image littérale et ensuite dans l'accès au sens figuré. Nous avons pu remarquer que l'interprétation des métaphores est aussi un facteur qui est influencé par la connotation sémantique octroyée aux métaphores de la L1 ; celles-ci peuvent amener l'apprenant vers une interprétation appropriée de l'EI de la L2, mais aussi conduisent-elles le locuteur non-natif à commettre des erreurs d'interférence négative, telles que nous allons les présenter dans l'analyse du corpus de notre recherche.

\section{Méthodologie}

Etant donné que l'aspect concernant l'enseignement-apprentissage des EI est un sujet fondamental à aborder afin de rendre le locuteur non-natif plus performant dans la L2, nous nous sommes intéressées plus particulièrement à l'influence de la L1 des apprenants lors de la compréhension et de la production des EI de la L2.

Pour ce faire, nous allons implémenter dans ce travail une approche méthodologique mixte en associant à la fois des éléments de la recherche qualitative et quantitative. Dans cette perspective, les éléments de l'approche qualitative nous permettront d'interpréter, d'associer et d'expliquer les statistiques fournies par l'approche quantitative, ce qui nous aidera à valider le processus du travail de recherche de manière plus précise et détaillée. De plus, puisque nous avons élaboré des tests de compréhension et de production des EI, une approche mixte sera développée qui analysera en priorité la description et l'explication ainsi que la représentativité des données.

De la même manière, nous avons ciblé deux publics afin d'obtenir des données à la fois différentes et complémentaires sur un même sujet (le rôle de la L1 dans la compréhension et la production des EI de la L2). De cette façon nous avons compris et analysé les stratégies adoptées par les locuteurs colombiens et les hispanophones vivant à Toulouse en compréhension et en production des EI ; ainsi, cette méthode nous a permis de mieux comprendre notre problématique de recherche et nous a aussi offert l'occasion de fixer les objectifs de notre travail.

\section{Objectif général}

L'étude expérimentale que nous allons proposer doit permettre de déterminer dans quelle mesure les apprenants emploient la connaissance de leur L1 afin de comprendre et de produire les ei de la L2. Pour ce faire, nous avons élaboré 4 tests : deux de production, un d'acceptabilité et un de compréhension afin de tester deux publics différents : des hispanophones 
vivant à Toulouse (milieu hétéroglotte) et des hispanophones de la Colombie (milieu homoglotte).

\section{Questions de recherche et hypothèses}

La présente étude concerne l'influence de la L1 (espagnol) dans la compréhension et la production des EI de la L2 (français). Comme nous l'avons expliqué au préalable, les locuteurs non-natifs ont tendance à recourir à leur L1 afin d'activer un réseau d'associations qui leur permet de pallier la méconnaissance de certaines structures figées de la L2. Cette stratégie contribue à déclencher d'une part des transferts positifs qui peuvent aider l'apprenant à accéder plus rapidement au sens sous-jacent de l'expression, mais aussi des transferts négatifs qui affectent, au niveau sémantique et lexical, la compréhension et la production de ces tournures de la L2. En ce sens, en suivant l'objectif décrit précédemment, nous posons les questions suivantes :

1. Dans quelle mesure les apprenants utilisent la connaissance de leur L1 afin de comprendre et de produire les EI de la L2 ?

2. Le type de l'EI joue-t-il un rôle dans la compréhension et la production de celle-ci?

3. Quelles stratégies sont mises en oeuvre par les apprenants afin de produire des EI plus ou moins connues?

Concernant la première question, les apprenants étrangers ont recours à la L1 plus généralement quand les deux systèmes linguistiques appartiennent à la même famille. Par conséquent, il y a des ressemblances entre la L1 et L2 aux niveaux sémantique, métaphorique, iconique et lexical. C'est le cas, par exemple, de l'espagnol et du français (Irujo 292 ; Boers et Demecheleer 257 ; Detry, Estrategies memorísticas 72). Néanmoins, les apprenants colombiens auront tendance à recourir davantage à leur L1 lors de la production et de la compréhension, alors que les hispanophones vivant à Toulouse y recourent afin de comprendre l'EI. En production, ces derniers auront tendance à s'éloigner de l'équivalence lexicale entre les deux langues afin d'essayer de trouver un équivalent sémantique dans la L2. Par exemple, être dans la lune est une tournure identique aux deux langues, mais les apprenants privilégieraient la formule avoir la tête en l'air plutôt que de transférer les constitutifs de l'expression de la L1, même si cette tournure est valable en L2 ; car, ils emploient des stratégies d'ordre intralinguistique.
En ce qui concerne la deuxième question, nous considérons que le degré de connexion entre la L1 et la L2 influence l'apprentissage des tournures idiomatiques. En ce sens, dans les trois catégories proposées, il y aurait des transferts positifs ou négatifs. Ainsi, les EI identiques et similaires seront comprises plus facilement que les EI différentes qui présentent un degré d'opacité sémantique très élevé, ce qui ne permet pas aux apprenants de faire des associations avec leur L1.

De la même manière, les EI similaires sont susceptibles de présenter plus de cas d'interférences (ou transferts négatifs) lorsqu'il s'agit de la production, car les apprenants auraient tendance de transposer les éléments constitutifs de l'EI de la L1 vers la L2. Par exemple, le non-natif favoriserait l'expression parler entre les dents (traduction littérale de l'EI espagnole hablar entre los dientes) plutôt que la tournure parler dans sa barbe. La catégorie des expressions similaires viendrait ainsi nuancer l'hypothèse du transfert et ce, notamment en termes d'opacité sémantique, de fréquence, de structure et de lexique.

Quant aux EI différentes, elles restent plus complexes à comprendre et à produire, car la relation entre les constitutifs de la tournure et le sens sousjacent est arbitraire et l'image littérale de l'EI de la L2 ne correspond pas à celle de la L1 (par exemple : se payer la tête de quelqu'un / tomarle el pelo a alguien).

Finalement, concernant la troisième question, il est probable que les apprenants vivant à Toulouse aient tendance à considérer les EI identiques non transférables à la L2 (Kellerman 70). Ils mettent ainsi en œuvre des stratégies d'ordre intralinguistique. Ils pourraient aussi employer, dans la production des EI différentes, la définition sémantique pour pallier l'oubli des éléments constitutifs de l'expression de la L2 ; par exemple, l'apprenant dirait dormir profondément au lieu d'employer la tournure idiomatique dormir à poings fermés. En ce qui concerne les apprenants colombiens, ils emploieraient des stratégies d'ordre interlinguistique en faisant des associations toujours avec la L1. Pour cette raison, ils s'exposeraient à plus de transferts négatifs lors de l'utilisation des EI similaires et différentes (Detry, En luttre 127).

\section{Méthode de recherche}

\section{Participants :}

Etant donné la complexité des EI aussi bien en compréhension qu'en production, nous avons décidé de faire passer les tests aux apprenants de niveau avancé 
en français (L2). En ce sens, nous avons sondé 26 apprenants colombiens (public A) de niveau B2 et 26 hispanophones Latino-Américains vivant à Toulouse (public B) de niveau C1. Grâce à ces deux publics, il nous sera permis d'analyser la performance au niveau linguistique et les stratégies mises en place lors de la compréhension et la production des EI françaises.

En ce qui concerne les caractéristiques des participants, le public A est composé d'étudiants de la Licence en langues à la Universidad de Pamplona, qui ont entre 20 et 25 ans et qui ont suivi des cours de français depuis 4 ans, à raison de 5 heures par semaine. Concernant le public B, il s'agit d'étudiants de master et de doctorat, qui, avant de venir en France ont étudié le français pendant 4 à 5 ans approximativement. Ils ont entre 25 et 32 ans, et sont en France depuis 1 ou 2 ans généralement.

\section{Procédure de sélection des EI :}

Afin d'accomplir les objectifs de notre travail, nous avons sélectionné 30 EI françaises en les classant en trois catégories :

1. EI identiques avec équivalence lexicale et sémantique entre les deux langues

2. EI similaires avec équivalence sémantique, mais avec une "petite divergence" lexicale

3. EI différentes avec équivalence sémantique, mais de composition lexicale totalement différente.

En ce sens, nous avons trouvé 10 tournures correspondant à chaque catégorie. A partir de ce premier classement, nous avons demandé de l'aide à des locuteurs natifs afin de déterminer si effectivement ils emploient ces EI de manière habituelle et dans quel contexte d'énonciation.

Ainsi, nous avons effectué la sélection des EI selon les paramètres décrits au préalable. Néanmoins, en ce qui concerne l'élaboration de la contextualisation des EI, nous avons testé les étudiants francophones du master 2 FLE à l'Université Toulouse II Jean-Jaurès ; ils devaient donc fournir l'EI selon la situation d'énonciation décrite. Ce prétest nous a permis de vérifier l'efficacité de la description du contexte afin de déclencher chez l'apprenant l'Ei et aussi de revérifier si la tournure était connue par les locuteurs natifs. Etant donné les résultats de ce test, nous avons décidé de réélaborer le contexte de la tournure et de changer certaines EI. Après ce processus de sélection et de vérification, nous avons décidé de consolider les trois catégories des EI pour nous pencher enfin sur l'élaboration des tests.

\section{Les expressions idiomatiques testées $(\mathrm{N}=30)$}

Les tableaux ci-dessous exposent clairement les EI testées auprès des étudiants. Le premier tableau présente les EI identiques entre les deux langues; le tableau 2 expose les EI similaires entre les deux systèmes linguistiques et nous avons fait la traduction littérale en français afin de remarquer le changement de l'image de l'expression en espagnol. Le tableau 3 remarque les EI complétements différentes entre les deux langues ; de même nous avons fait la respective traduction littérale en français afin que le lecteur puisse comprendre le changement de l'image de chaque expression.

Tableau 1. EI identiques

\begin{tabular}{ll}
\hline Etre dans la lune & Estar en la luna \\
\hline Avoir un mot sur le bout de la langue & Tener una palabra en la punta de la lengua \\
\hline Prendre le taureau par les cornes & Agarrar el toro por los cuernos \\
\hline Entrer par une oreille et sortir par l’autre & Entrar por una oreja y salir por la otra \\
\hline Se noyer dans un verre d'eau & Ahogarse en un vaso de agua \\
\hline Ne pas rester les bras croisés & No quedarse con los brazos cruzados \\
\hline Etre mort de rire & Estar muerto de risa \\
\hline C'est son talon d'Achille & Es su talón de Aquiles \\
\hline Tuer la poule aux œufs d’or & Matar la gallina de los huevos de oro \\
\hline Avoir bon cœur & Tener buen corazón \\
\hline
\end{tabular}

Source : Elaboration propre 
Tableau 2. EI similaires

\begin{tabular}{ll}
\hline Coûter les yeux de la tête & Costar un ojo de la cara (« coûter un œil du visage ») \\
\hline Chercher midi à quatorze heures & Buscarle cinco patas al gato (« chercher cinq pattes au chat ») \\
\hline Parler dans sa barbe & Hablar entre los dientes (« parler entre les dents ») \\
\hline Dormir les poings fermés & Dormir a pierna suelta (« dormir à jambe relâchée ») \\
\hline Payer les pots cassés & Pagar los platos rotos ( payer les assiettes cassées ») \\
\hline Jeter de l’huile sur le feu & Echarle leña al fuego ( jeter du bois au feu ») \\
\hline Avoir le moral à zéro & Tener la moral por el piso (« avoir le moral au sol ») \\
\hline Etre de mauvais poil & Estar de malas pulgas (« être de mauvaises puces ») \\
\hline Enlever une épine du pied & Quitarle un peso de encima (« enlever un poids du dessus ») \\
\hline Ne pas avoir la langue dans sa poche & No tener pelos en la lengua (« ne pas avoir des poils sur la langue) \\
\hline
\end{tabular}

Source : Elaboration propre

Tableau 3. EI différentes

\begin{tabular}{ll}
\hline Casser sa pipe & Colgar los guayos («suspendre les chaussures de sport ») \\
\hline Poser un lapin & Dejar plantado alguien (« laisser quelqu’un planté ») \\
\hline Avoir le coup de foudre & Quedar flechado ( rester fléché ») \\
\hline Tenir la chandelle & Tocar el violín (« toucher le violon ») \\
\hline Faire d’une pierre deux coups & Matar dos pájaros de un tiro (« tuer deux oiseaux d'un même tir ») \\
\hline En avoir ras le bol & Estoy hasta la coronilla ( être jusqu'au sommet du crâne ») \\
\hline Il pleut des cordes & Cae un palo de agua (« il tombe un bâton d’eau ») \\
\hline Il y a anguille sous roche & Hay gato encerrado (« il y a chat enfermé ») \\
\hline Etre un moulin à paroles & Hablar hasta por los codos (« parler même par les coudes ») \\
\hline Se payer la tête de quelqu’un & Tomarle el pelo ( prendre le poil à quelqu'un ») \\
\hline
\end{tabular}

Source : Elaboration propre

\section{Batterie de tests :}

Afin d'éprouver les apprenants en production et en compréhension des EI, quatre tests ont été élaborés : deux de production, un d'accéptabilité et un de compréhension. Tout d'abord, nous avons créé un contexte pour chaque EI afin d'activer chez l'apprenant l'idiomaticité de l'énonciation, ce qui permettrait de déclencher plus facilement l'EI. Les tests ont été administrés à des locuteurs français avant de sonder les locuteurs non natifs. Cette procédure nous a permis de vérifier d'une part si la contextualisation de la tournure a été bien construite et d'autre part si les locuteurs natifs utilisent la tournure dans le contexte décrit.

Dans tous les tests, les EI étaient mélangées et elles n'étaient pas présentées selon la catégorie, ainsi que les trente EI ont été testés quatre fois dans les quatre tests différents. De la même manière, les quatre tests ont été administrés par ordre décroissante de difficulté, à savoir : test de production (test 1), test d'acceptabilité (test 2), test de production avec traduction (test 3) et test de compréhension (test 4).

Concernant le premier test de production (test 1), nous avons distribué les trois catégories d'Ei selon un ordre différent. Par conséquent, l'apprenant pouvait trouver tout d'abord une expression différente, puis une identique et ensuite une similaire. Pour ce test, nous avons élaboré les énoncés en français et nous avons inclus une partie de l'EI. Les apprenants devaient donc fournir le mot manquant pour compléter la tournure française.

Nous considérons que ce test est le plus complexe pour les apprenants car il demande non seulement de comprendre l'ensemble du lexique employé pour la description du contexte et, de plus, il exige 
de connaître la tournure idiomatique, de la récupérer et de la produire. A cause de ces facteurs qui exigent un coût cognitif très élevé, le test de production (test 3) a été élaboré. Cette fois, nous avons délibérément apposé la contextualisation de l'expression en espagnol et en français afin de nous assurer que l'apprenant ait bien compris l'ensemble du lexique de la description du contexte. Le test est donc composé d'un énoncé en espagnol contenant l'EI et d'une traduction en français avec l'EI omise.

En ce qui concerne le test d'acceptabilité (test 2), nous l'avons élaboré car nous voulions vérifier si les apprenants reconnaissaient et acceptaient, ou par contre s'ils refusaient les images formées par les EI de leur L1 celles-ci transposées vers le français. Nous avons choisi les 30 EI espagnoles et nous les avons traduites de manière littérale en français. Les apprenants devaient ainsi répondre par oui ou par non à la question de savoir si les EI traduites littéralement se disaient en français.

Concernant le test de compréhension (test 4), nous l'avons élaboré afin de savoir si les apprenants connaissaient le sens de l'EI. Les apprenants devaient donc relier chaque tournure à son sens en choisissant parmi quatre options. Nous avons en ce sens conçu la bonne réponse et trois distracteurs pour chaque expression selon les paramètres suivants : la bonne réponse était une paraphrase de l'EI testée, le premier distracteur était une paraphrase avec une "petite divergence ", le deuxième distracteur était l'interprétation littérale de l'EI testée, enfin le troisième distracteur était un item sans rapport aucun avec l'EI testée.

Enfin, nous avons décidé que l'administration des 4 tests devait se dérouler le même jour et en suivant l'ordre consécutif décrit plus haut. Chaque apprenant a complété les tests seul, sans l'aide de son enseignant ou de ses camarades et sans l'aide non plus du dictionnaire ou autre ressource documentaire. Nous n'avons pas imposé une limite de temps pour répondre à chaque test.

\section{Résultats}

D’après les résultats et l'analyse de chaque test, nous pouvons remarquer que les sujets ont recours à la L1 généralement quand il existe des ressemblances entre les deux systèmes linguistiques (Irujo 295 ; Boers et Demecheleer 258 ; Detry, Estrategias memorísticas 178). Selon l'analyse intragroupe, nous constatons que les sujets du niveau B2 sont plus attachés à la production de tournures de la L2 où l'iconicité est très transparente à celle de la L1; c'est pour cette raison que nous n'avons pas trouvé dans l'analyse de données des stratégies de type intralinguistique. Par ailleurs, le niveau $\mathrm{C} 1$ préfère produire des expressions très opaques entre la $\mathrm{L} 1$ et la $\mathrm{L} 2$, comme par exemple avoir le moral dans les chaussettes au lieu d'avoir le moral à zéro, tournure plus transparente à l'expression espagnole tener la moral por el piso (trad. Avoir le moral au sol). En ce sens, nous pouvons affirmer la validité de notre première hypothèse en disant que les apprenants de niveau avancé, dans ce cas-ci le niveau $\mathrm{C} 1$, ont tendance à utiliser des stratégies intralinguistiques pour essayer de produire et de comprendre les EI de la L2.

Dans cette optique, nous avons également constaté que le type d'Eı joue un rôle primordial dans la production et la compréhension des tournures, comme Irujo l'a proposé dans son étude avec des apprenants vénézuéliens de niveau avancé d'anglais L2 (285). En effet, nos hypothèses émises à ce propos sont valables pour le niveau B2, car nous avons observé que les apprenants colombiens, dans l'analyse des réponses correctes, sont plus performants dans la production et la compréhension des expressions identiques et similaires ; en revanche, ils présentent plus de difficultés au moment de produire et de comprendre les EI qui sont totalement différentes dans les deux langues.

Néanmoins, nos hypothèses divergent en ce qui concerne les résultats du niveau $\mathrm{C} 1$, car nous remarquons que les sujets testés ont tendance à produire et à comprendre plus facilement les expressions identiques et les tournures différentes, alors que la catégorie d'EI similaires engendre des difficultés dans les deux compétences. Nous pouvons, donc, supposer que la formation iconique déjà acquise des structures figées de la L2 est entravée par les différences plus subtiles qui existent entre la L1 et la L2. Ces résultats nous pouvons les constater dans le tableau 4 où nous présentons les résultats des tests concernant les réponses correctes de chaque groupe testé.

De plus, en ce qui concerne les stratégies mises en place par les sujets afin de produire des EI, nous pouvons remarquer que les deux niveaux ont tendance à recourir à la L1 pour établir des associations interlinguistiques et ainsi pallier la méconnaissance des tournures de la L2. Dans cette perspective, nous pouvons souligner que, généralement, les apprenants produisent des transferts positifs et des transferts négatifs. D’après les résultats obtenus, nous avons souligné que les deux groupes testés ont produit des 
Tableau 4. Résultats des réponses correctes : niveau B2 et C1

\begin{tabular}{lcccccccc}
\hline Type dexpression idiomatique & $\begin{array}{c}\text { Test } 1 \\
\text { B2 }\end{array}$ & $\begin{array}{c}\text { Test } 1 \\
\text { C1 }\end{array}$ & $\begin{array}{c}\text { Test } 2 \\
\text { B2 }\end{array}$ & $\begin{array}{c}\text { Test 2 } \\
\text { C1 }\end{array}$ & $\begin{array}{c}\text { Test 3 } \\
\text { B2 }\end{array}$ & $\begin{array}{c}\text { Test 3 } \\
\text { C1 }\end{array}$ & $\begin{array}{c}\text { Test } 4 \\
\text { B2 }\end{array}$ & $\begin{array}{c}\text { Test } 4 \\
\text { C1 }\end{array}$ \\
\hline Identiques & & & & & & & & \\
\hline M & 6,76 & 7,73 & 9,30 & 9,19 & 7,76 & 8,07 & 8,53 & 8,96 \\
Ecart-type & 1,33 & 1,25 & 0,88 & 0,98 & 1,63 & 1,87 & 1,10 & 0,82 \\
\hline Similaires & & & & & & & & \\
\hline M & 1,03 & 2,53 & 4,61 & 5,38 & 0,69 & 1,46 & 6,96 & 8,15 \\
Ecart-type & 0,95 & 2,78 & 1,72 & 2,07 & 0,78 & 2,06 & 0,99 & 1,04 \\
\hline Différentes & & & & & & & & \\
\hline M & 0,23 & 3,26 & 5,92 & 7,23 & 0,38 & 2,92 & 6,34 & 8,42 \\
Ecart-type & 0,51 & 3,00 & 2,15 & 1,72 & 0,57 & 2,74 & 2,20 & 1,65 \\
\hline
\end{tabular}

Source : Elaboration propre

transferts positifs dans la catégorie d'EI identiques, plus particulièrement sur trois tournures : c'est son talon d'Achille, être mort de rire et l'avoir sur le bout de la langue.

Quant aux transferts négatifs, les deux niveaux testés ont tendance à en produire dans la catégorie d'EI similaires et différentes ; cependant nous remarquons, dans le tableau 5, que le niveau B2 est plus enclin à ce genre de transferts que le niveau $\mathrm{C} 1$.

D’après ces résultats, nous constatons que les expressions similaires qui sont sujettes à avoir des transferts négatifs sont celles qui ont un ou deux composants lexicaux divergeant entre les deux langues. Par exemple, notons les expressions parler dans sa barbe / hablar entre los dientes (trad. Parler entre les dents), avoir le moral à zéro / tener la moral por el piso (trad. Avoir le moral au sol), jeter de l'huile sur le feu / echarle leña al fuego (trad. Jeter du bois au feu). Par ailleurs, lorsque les sujets rencontrent des tournures similaires qui coïncident dans les deux langues avec un seul composant lexical, ils optent pour la stratégie de l'évitement. Les sujets semblent en effet incapables de faire des associations interlinguistiques, ne donnant souvent aucune réponse ou bien la fréquence des réponses correctes et des réponses transferts n'est pas assez considérable. C'est le cas de chercher midi à quatorze heures / buscarle cinco patas al gato (trad. Chercher cinq pattes au chat) et dormir à poings fermés / dormir a pierna suelta (trad. Dormir à jambe relâchée).

Concernant les transferts négatifs dans les expressions différentes, nous avons remarqué que cette catégorie a une fréquence de transferts négatifs qui n'est pas supérieure à celle de la catégorie

Tableau 5. Résultats des réponses transfert : niveau B2 et C1

\begin{tabular}{|c|c|c|c|c|c|c|}
\hline Type d'expression idiomatique & $\begin{array}{c}\text { Test } 1 \\
\text { B2 }\end{array}$ & $\begin{array}{c}\text { Test } 1 \\
\text { C1 }\end{array}$ & $\begin{array}{c}\text { Test } 2 \\
\text { B2 }\end{array}$ & $\begin{array}{c}\text { Test } 2 \\
\text { C1 }\end{array}$ & $\begin{array}{c}\text { Test } 3 \\
\text { B2 }\end{array}$ & $\begin{array}{c}\text { Test } 3 \\
\text { C1 }\end{array}$ \\
\hline \multicolumn{7}{|l|}{ Identiques } \\
\hline M & 1,19 & 0,80 & 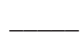 & 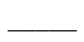 & 0,38 & 0,50 \\
\hline Ecart-type & 0,69 & 0,80 & $\underline{-}$ & $\underline{-}$ & 0,49 & 0,64 \\
\hline \multicolumn{7}{|l|}{ Similaires } \\
\hline M & 3,07 & 2,38 & 5,30 & 4,53 & 5,73 & 4,50 \\
\hline Ecart-type & 1,29 & 1,32 & 1,73 & 2,02 & 1,66 & 1,79 \\
\hline \multicolumn{7}{|l|}{ Différentes } \\
\hline M & 1,23 & 0,46 & 4,00 & 2,73 & 4,34 & 1,34 \\
\hline Ecart-type & 1,03 & 0,64 & 2,22 & 1,68 & 1,99 & 1,38 \\
\hline
\end{tabular}


d'expressions similaires. D'après l'analyse contrastive, le niveau B2 n'a pas des fréquences considérables de réponses correctes dans cette catégorie d'EI différentes, mais montre une hausse considérable de transferts négatifs. Nous en déduisons que les sujets transposent les composants lexicaux de la L1 vers la L2 lorsqu'ils ne sont pas familiarisés avec ce genre de tournures; c'est le cas en général pour les expressions tenir la chandelle, faire d'une pierre deux coups et il pleut de cordes qui sont susceptibles d'avoir plus de cas de transferts négatifs. De plus, nous avons déterminé que les expressions qui n'ont pas de réponses correctes et une fréquence minime de réponses transferts sont inconnues pour les sujets testés, c'est le cas des tournures ily a anguille sous roche, être un moulin à paroles, casser sa pipe et en avoir ras le bol.

En revanche, le niveau $\mathrm{C} 1$ présente dans la catégorie d'EI différentes plus de cas de réponses correctes que de transferts négatifs. Nous pouvons remarquer que les tournures qui ont une fréquence élevée de réponses correctes n'ont pas de cas de transferts, c'est le cas d'avoir le coup de foudre, en avoir ras le bol et il pleut de cordes. En revanche, les tournures qui ont présenté une complexité majeure au moment de la production sont celles qui n'ont pas une fréquence élevée de réponses correctes ni de réponses transferts, c'est le cas de casser sa pipe, se payer la tête de quelqu'un, il y a anguille sous roche et être un moulin à paroles.

D'après cette analyse, nous constatons que la compréhension et la production des EI (identiques, similaires et différentes) sont liées à la familiarité, à la fréquence d'emploi et à la performance linguistique de l'apprenant (Abel 340 ; Laufer 189). Nous déduisons que les hispanophones vivant à Toulouse ont tendance à employer les EI différentes, car ils sont en contact quotidiennement avec les locuteurs natifs, ce qui leur permet d'associer l'emploi d'une expression opaque avec une situation réelle de communication où les locuteurs natifs utilisent une tournure déterminée. Par contre, les Colombiens ne récupèrent pas facilement ce genre de tournures, car ils n'emploient pas ces types d'expressions dans leur vie quotidienne ni n'ont l'occasion d'être en contact avec les locuteurs natifs.

En ce sens, nous pouvons mettre l'accent sur trois paramètres qui influencent la production et la compréhension des ei de la L2. Tout d'abord, le degré de transparence ou d'opacité d'une tournure ; ensuite, la familiarité et la fréquence d'emploi des tournures idiomatiques sont des caractéristiques qui facilitent ou empêchent la compréhension ou le traitement d'inférences chez les locuteurs non-natifs. Enfin, l'accès au sens figuré des EI dépend énormément de l'exposition de l'apprenant pour bien comprendre la relation étroite entre le langage, la culture et les métaphores, facteurs qui sont véhiculés par les EI de la L2 et qui sont interprétés en faisant des associations interlinguistiques.

A cet égard, d'après les trois analyses mises en place (analyses intragroupe, intergroupe et supragroupe), nous constatons que l'influence de la L1 joue un rôle primordial dans la production et la compréhension d'EI d'une L2, car il semble que les stratégies interlinguistiques servent à élaborer un ensemble de représentations entre les deux langues, plus particulièrement, des représentations métalinguistiques et des représentations culturelles, lesquelles sont généralement sédimentées à partir du substrat de la L1 afin de produire et de comprendre plus facilement les tournures de la L2. Ainsi, d'un point de vue didactique, il est clair que la L1 doit être considérée comme un point d'ancrage dans l'accès idiomatique d'une L2 (Detry «En lutte contre l'arbitraire »; Estrategias memorísticas).

\section{Limites et perspectives possibles d'étude}

Au vu des résultats de notre étude, nous précisons ici quelques limites et possibles perspectives de travaux de recherche dans le champ de la phraséodidactique.

En premier lieu, nous avons abordé trois catégories d'EI (identiques, similaires et différentes) afin de savoir laquelle présente le plus de difficultés au moment de produire et de comprendre les EI de la L2. Ainsi nous voulions déterminer quelle catégorie de tournures est la plus susceptible d'avoir de cas de transferts positifs et de transferts négatifs. Néanmoins, nous n'avons pas traité une quatrième catégorie d'Eı proposée par Laufer : il s'agit de celle des tournures de la L2 qui ne trouvent pas des équivalents idiomatiques dans la L1 ou vice versa. De même, nous n'avons pas abordé la catégorie des tournures qui comprennent la forme lexicale identique dans la L1 et la L2, mais qui présentent des divergences sémantiques dans les deux langues. Par conséquent, à partir des résultats de cette étude, nous aurions l'occasion d'envisager une analyse concernant les cinq catégories possibles de tournures idiomatiques, ce qui contribuerait de manière efficace à discerner certaines pistes de l'enseignement-apprentissage des EI 
d'une langue étrangère, plus particulièrement chez les hispanophones.

Par ailleurs, nous avons analysé les tournures qui ont eu une fréquence significative de réponses correctes ou de réponses transferts, bien que nous n'ayons pas établi les degrés d'opacité ou de transparence que possède chaque expression, même si celle-ci appartient à la catégorie d'EI similaires. En effet, lors de notre analyse, nous avons découvert que chaque tournure testée a des degrés d'opacité dans les trois catégories, mais nous n’avons pas érigé, au début de notre recherche, des paramètres pour évaluer cet aspect qui nous paraît à l'heure actuelle très important au moment de justifier les réponses données par les sujets et au moment de discerner quelle tournure est moins ou plus complexe à produire ou à comprendre en considérant chaque catégorie.

En outre, nous avons choisi deux groupes de différents milieux : des hispanophones de la Colombie (Niveau B2) et des hispanophones vivant à Toulouse (Niveau C1), ce qui nous a permis de signaler l'importance du contexte d'immersion afin de se familiariser avec les EI différentes qui ont présenté des difficultés considérables pour le niveau B2. Cependant, il serait intéressant d'adapter cette méthode de recherche aux apprenants colombiens d'un niveau B1 et B2 afin d'analyser les différentes stratégies mises en place pour pallier la méconnaissance des EI de la L2. De sorte que nous pourrions mettre en place une méthodologie adaptée aux besoins des apprenants au niveau de la compréhension et de la production des tournures idiomatiques de la L2.

Ainsi, nous pensons que la présente étude portant sur un public spécifique, peut être reprise dans l'analyse de la production et de la compréhension d'EI dans le cas des autres apprenants non-natifs, et pas seulement dans le cadre de l'enseignement/apprentissage du FLE chez les hispanophones. Ce facteur permettrait de mettre l'accent sur la diversité culturelle, ainsi que sur l'influence notoire de la L1 dans l'acquisition d'une L2.

\section{Conclusion}

Les EI sont au cœur du système de communication, elles sont omniprésentes dans le discours quotidien d'un locuteur natif, car elles constituent une des bases de la sagesse culturelle. Ainsi permettent-elles aux apprenants étrangers une interaction réelle entre la langue et la culture. De même, le caractère figé des éléments constitutifs permet de motiver l'apprenant à l'économie linguistique, ce qui témoignerait d'une bonne maîtrise de la L2 au niveau du lexique, de la syntaxe, de la sémantique et de la pragmatique. Pour cela, l'apprentissage de ces tournures entraîne l'apprenant à devenir capable d'interpréter le sens métaphorique dans le discours en s'appuyant sur les connaissances extralinguistiques afin d'être plus autonome dans l'acte communicatif.

En conséquence, la catégorisation des EI (identiques, similaires et différentes) par rapport au degré d'opacité ou de transparence en L2 et à l'équivalence en L1 pourrait favoriser considérablement la rétention en mémoire des composants lexicaux d'une tournure donnée. En effet, le travail centré sur l'analyse des ressemblances et des dissemblances lexicales et sémantiques s'avère un composant indispensable pour renforcer le processus de maîtrise du caractère métaphorique de l'EI.

Dans ce processus interlinguistique, l'apprenant pourrait accéder à la culture étrangère en réfléchissant parallèlement sur sa propre culture, ce qui l'aiderait à devenir plus compétent dans l'appréciation des référents idiomatiques au sein de la L2. Autrement dit, le but de l'acquisition d'une L2 est lié au besoin d'imprégner l'apprenant à la richesse de ses tournures façonnées par la transmission d'un héritage socio-linguistique qui mérite notre attention dans l'élaboration d'unités didactiques et la mise en place dans des scénarios pédagogiques.

En effet, la maîtrise des EI recouvre un vaste champ de compétences, allant de la compétence communicative et socioculturelle à la compétence pragmatique qui permet à l'apprenant l'appropriation d'éléments en situation pour déduire la signification sous-jacente que véhicule une tournure. Donc, en cours d'une L2, il est nécessaire d'assurer un emploi correct des EI en proposant des activités de sensibilisation et d'inférence, c'est-à-dire, un travail d'interprétation du contexte d'énonciation suivi d'une compréhension de ce que dit l'interlocuteur ainsi que ses intentions, ses attitudes et ses insinuations.

Dans cette perspective, la place des EI doit être considérée dès les premiers niveaux d'apprentissage d'une L2. Le but serait de parvenir à mieux sensibiliser les apprenants aux procédés d'emploi de ces tournures signifiantes. Ces derniers auraient alors l'occasion de se rendre compte des différences et similitudes lexicales entre la L2 et celles de la L1, ce qui leur permettrait de distinguer des relations existantes au niveau sémantique, ainsi que d'évoquer un contraste d'ordre culturel. En ce sens, le processus 
d'acquisition permet d'explorer au maximum l'aspect interculturel des expressions favorisant un système cohérent dans l'apprentissage, de tendre par ce biais à ce que les savoirs deviennent des savoir-faire.

D’après nos résultats, les apprenants hispanophones trouvent moins complexe la compréhension que la production des EI. Pour cette raison, au moment où les apprenants emploient une tournure, ils doivent tenir compte du caractère stable et invariable de chaque constituant. Cette spécificité des EI ne peut pas être laissée de côté dans l'enseignement des langues. Au contraire, elle doit être abordée en considérant le niveau des apprenants, c'est-à-dire que l'enseignant doit choisir les EI selon leur degré d'opacité en tenant compte des compétences des apprenants et aussi des équivalents dans la L1.

De ce point de vue, nous estimons que les EI identiques doivent être abordées dès les premiers niveaux de langues. Alors qu'à partir d'une certaine maîtrise lexicale, il faut mettre en place des EI similaires et différentes, de telle manière que les stratégies d'enseignement soient centrées sur ces deux dernières catégories, plus particulièrement sur la catégorie d'EI similaires, car les apprenants ont tendance à transposer totalement les éléments lexicaux de la L1 vers la L2. En ce sens, l'objectif d'une méthodologie pour l'acquisition des EI est celui de reconnaître les propriétés spécifiques des tournures de la L2 et de les envisager autour d'un travail d'associations entre forme et sens, ce qui permettrait à l'apprenant tant de mettre en relation la signification de l'ensemble de constitutifs énoncés que de produire une EI qui convient le mieux au contexte d'énonciation. De plus, la sélection d'une tournure adaptée au contexte d'énonciation exige de la part de l'apprenant à développer des habiletés cognitives en faisant des associations des connaissances ou des concepts acquis au cours de sa L1 ou dans d'autres langues connues pour les mettre en relation avec les nouvelles images de l'EI en L2 et arriver enfin à dévoiler le sens figuré.

De fait, nous pouvons souligner que ce type d'associations interlinguistiques et aussi intralinguistiques joue un rôle prépondérant dans le processus de rétention et d'emploi des EI. Cela représente aussi des stratégies d'apprentissage activées par l'apprenant pour mieux décoder l'iconicité de la tournure et, ensuite, mieux lui permettre l'accès au dévoilement du caractère idiomatique.

Par ailleurs, il est nécessaire de faire remarquer l'importance de l'acquisition et la maîtrise d'un répertoire lexical très vaste dans tous les domaines, ce qui permettrait à l'apprenant d'interpréter et d'accéder plus facilement au sens sous-jacent des tournures de la L2. Dans cette perspective, lorsque une EI est constituée d'un nombre équivalent d'éléments lexicaux dans les deux langues, la probabilité pour que les apprenants étrangers accèdent à la décodification de la tournure est plus haute. Mais si les apprenants ne trouvent pas une équivalence dans les deux langues, ils vont hésiter, dans une première approximation, sur la signification de l'expression étrangère.

En ce sens, la compréhension lexicale des EI, la visualisation mentale de l'image que motive le sens figuré, la prise de conscience d'une lecture qui dépasse le niveau explicite de la signification de chaque constituant, sont des paramètres obligatoires dans le processus cognitif d'assimilation et ensuite de réflexion et d'interprétation de la valeur iconique des tournures.

De plus, il faut souligner aussi que le contact que les apprenants ont avec des locuteurs natifs contribue à développer des capacités d'associations et à établir des paramètres des structures figées de la langue. Pour cette raison, nous avons observé dans l'analyse des résultats de notre étude que les apprenants hispanophones vivant à Toulouse présentent moins de difficultés dans la production et la compréhension d'EI différentes que les apprenants colombiens qui ne sont pas en contact direct avec des natifs. Ainsi, le facteur de fréquence et de familiarité avec ce genre de tournures figées leur permettent de mieux reconnaître le sens indépendant de chaque élément constitutif, mais aussi de comprendre le sens nouveau de la somme des constitutifs de l'expression de la L2.

En somme, notre étude nous a permis de considérer que les propositions didactiques devraient partir de ce qui est connu par les apprenants pour, au fur et à mesure de leurs processus d'acquisition de la L2, aborder les particularités ou les différences entre la L1 et la L2. De même, le recours à la L1 permet de valoriser la richesse des apprenants, tantôt au niveau culturel, tantôt au niveau pragmatique. Pour cette raison, il faut considérer l'influence de la L1 comme un facteur inhérent au processus d'acquisition d'une L2, notamment en ce qui concerne l'appréhension des tournures idiomatiques. Car, la relation entre la L1 et la L2 s'établit en élaborant un ensemble de représentations entre les deux langues, plus particulièrement, des représentations métalinguistiques et des représentations culturelles, généralement sédimentées à partir du substrat de la L1. 
Enfin, nous espérons avoir contribué à la recherche en didactique des langues mais aussi à celle de la phraséodidactique en proposant une dimension de recherche portant sur les EI de la L2 et aussi sur l'influence de la L1 (transfert positif et transfert négatif) en tant que stratégie cognitive naturelle qui est présente lors de l'acquisition d'une langue étrangère.

\section{Bibliographie}

Abel, Beate. "English idioms in the first language and second language lexicon: a dual representation approach." Second Language Research, vol. 19, no. 4, 2003, pp. 329358.

Bally, Charles. Traité de Stylistique française. 2e éd., Gallica Bibliothèque Numérique, 1921.

Bolly, Catherine. Phraséologie et collocations approche sur corpus en français $L 1$ et L2. Editions Scientifiques Internationales, 2011.

Boers, Frank, et Murielle Demecheleer. "Measuring the impact of crosscultural diferences on learners' comprehension of imageable idioms." ELT Journal, vol. 55, no. 3, 2001, pp. 255- 262.

Capra, Antonella. Les tournures idiomatiques dans le passage du français à l'italien et de l'italien au français. Thèse de doctorat, Université Toulouse II-Le Mirail/Università degli Studi di Parma, 2004.

Gibbs, Raymond et O’brien, Jennifer. "Idioms and mental imagery: The metaphorical motivation for idiomatic meaning." Cognition, no. 36, 1990, pp. 35-68.

Cummins, Jim, et Merrill Swain. Bilingualism in Education. Longman Press, 1986.

Detry, Florence. «En lutte contre l'arbitraire : pistes méthodologiques pour un apprentissage cognitif des expressions idiomatiques en LE ». Outils et méthodes d’apprentissage en phraséodidactique, édité par María Isabel Gonzalez Rey, EME Éditions, 2014, pp. 133-150.

Detry, Florence. Estrategias memorísticas y aprendizaje de las expresiones idiomáticas en lengua extranjera: el papel cognitivo de la iconicidad fraseológica. Thèse doctorale, Université de Girona, 2010.
Ellis, Rod. The study of second language acquisition. Oxford University press, 1994.

Gonzalez, Isabel. La phraséodidactique en action : les expressions figées comme objet d'enseignement. Universidad de Santiago de Compostela, 2008.

Gonzalez, Isabel. La phraséologie du français. Presses Universitaires du Mirail, 2002

Guilhermina, Jorge. "Les expressions idiomatiques correspondantes : analyse comparative ». Terminologie et Traduction, vols. 2-3, 1992, pp. 127-134.

Hoa, Thi. Enseignement des expressions figées métaphoriques françaises à des apprenants vietnamiens. Thèse de doctorat, Université de Montréal, 2009.

Irujo, Suzanne. "Don't put your leg in your mouth: transfer in the acquisition of idioms in a second language". $T E-$ SOL Quarterly, vol. 20, 1986, pp. 287-304.

Kellerman, Eric. "Towards the characterization of the strategy of transfer in second language learning". Interlanguage Studies Bulletin, vol. 2, 1986, pp. 58-145.

Laufer, Batia. "Avoidance of idioms in a second language: the effect of L1-L2 degree of similarity." Studia Linguistica, vol. 54, 2000, pp. 186-196.

Saussure, Ferdinand de. Cours de linguistique générale. Editions Payot et Rivages, 1995.

Théophanous, Olga. "Langage préfabriqué et développement de la fluidité verbale chez l'apprenant d'une L2 ». Innovations didactiques en français langue étrangère, édité par Claire Martinot et Anne Pégaz, Cellule de Recheche en Linguistique, 2014, pp. 117-128.

Théophanous, Olga. «Le traitement de la préconstruction lexicale dans un corpus des méthodes de français langue étrangère ». Enseigner le lexique, édité par Claudine Garcia-Debanc, Caroline Masseron et Christophe Ronveaux, Presses Universitaires de Namur, 2013, pp. 173-192. 